\title{
ARTICLE
}

Epidemiology and population health

\section{A Delphi study to build consensus on the definition and use of big data in obesity research}

\author{
Christina Vogel ${ }^{1} \cdot$ Stephen Zwolinsky ${ }^{2} \cdot$ Claire Griffiths $^{2} \cdot$ Matthew Hobbs $^{3} \cdot$ Emily Henderson $^{4} \cdot$ Emma Wilkins $^{2}$
}

Received: 31 May 2018 / Revised: 1 November 2018 / Accepted: 29 November 2018 / Published online: 17 January 2019

(c) The Author(s) 2019. This article is published with open access

\begin{abstract}
Background 'Big data' has great potential to help address the global health challenge of obesity. However, lack of clarity with regard to the definition of big data and frameworks for effectively using big data in the context of obesity research may be hindering progress. The aim of this study was to establish agreed approaches for the use of big data in obesity-related research.

Methods A Delphi method of consensus development was used, comprising three survey rounds. In Round 1, participants were asked to rate agreement/disagreement with 77 statements across seven domains relating to definitions of, and approaches to, using big data in the context of obesity research. Participants were also asked to contribute further ideas in relation to these topics, which were incorporated as new statements $(n=8)$ in Round 2. In Rounds 2 and 3 participants reappraised their ratings in view of the group consensus.

Results Ninety-six experts active in obesity-related research were invited to participate. Of these, 36/96 completed Round 1 (37.5\% response rate), 29/36 completed Round 2 (80.6\% response rate) and 26/29 completed Round 3 (89.7\% response rate). Consensus (defined as $>70 \%$ agreement) was achieved for $90.6 \%(n=77)$ of statements, with $100 \%$ consensus achieved for the Definition of Big Data, Data Governance, and Quality and Inference domains.

Conclusions Experts agreed that big data was more nuanced than the oft-cited definition of 'volume, variety and velocity', and includes quantitative, qualitative, observational or intervention data from a range of sources that have been collected for research or other purposes. Experts repeatedly called for third party action, for example to develop frameworks for reporting and ethics, to clarify data governance requirements, to support training and skill development and to facilitate sharing of big data. Further advocacy will be required to encourage organisations to adopt these roles.
\end{abstract}

These authors contributed equally: Christina Vogel, Stephen Zwolinsky

Supplementary information The online version of this article (https:// doi.org/10.1038/s41366-018-0313-9) contains supplementary material, which is available to authorized users.

Christina Vogel

cv@mrc.soton.ac.uk

1 MRC Lifecourse Epidemiology Unit, University of Southampton, SO16 6YD Southampton, UK

2 School of Sport, Leeds Beckett University, LS6 3QQ Leeds, UK

3 GeoHealth Laboratory, Geospatial Research Institute, University of Canterbury, Christchurch, New Zealand

4 Institute of Health \& Society, Newcastle University, Newcastleupon-Tyne NE1 4LP, UK

\section{Introduction}

Obesity is a persistent public health problem that no country has successfully addressed [1]. Novel datasets, particularly those not initially collected for obesity research, could provide important information to improve understanding of the interaction between, and relative influence of, the various determinants of obesity. Sources of continuously collected data have grown rapidly in recent years as a result of digitalised systems, and significant improvements in data processing and storage capabilities [2-4]. These large data sources are sometimes called 'big data'. The first two papers $[5,6]$ in this series demonstrate the increasing attention big data is garnering for obesity research and the wide variety of commercial and government data sources that are available and fit for purpose. They highlight the great potential big data has for formulating and evaluating policy, developing intervention initiatives for obesity 
prevention, and understanding its multiple determinants and their interactions. Nevertheless, big data in obesity and population research remains underutilised [7].

The slow adoption of big data in global efforts to reduce obesity prevalence may, in part, stem from a lack of clarity about the exact meaning of the term and what it entails for obesity-related research. Definitions can help describe the work needed and provide directions about associated skill, resource and infrastructure requirements [8]. There is no single, agreed definition of big data, yet it is often typified as being extensive in volume, derived from a wide variety of sources or collected at great velocity [2-4]. In the context of obesity research, the term big data often refers to novel data sets that have been collected for purposes other than health research, which may provide added value to more traditional data sources [3]. However, it has been debated whether traditional datasets, such as administratively collected medical records or large cohort studies, can also be deemed big data, particularly if they are linked to more novel data sources [4, 7, 9]. Reaching agreement on what big data encompasses in the context of obesity will help to increase precision and understanding of the term by researchers, and aid future activity in this field.

A clear definition is one that captures the meaning, use and function of a particular topic or concept, and guides researchers to develop a cohesive body of empirical evidence [10]. Clear definitions are valuable when developing research questions and presenting study findings because interpretations of loosely defined terms will be shaped by perceptions of the audience, who commonly have different educational, professional and cultural experiences [11]. Imprecise definitions can make it difficult to agree on what is being researched and may lead to studies examining disparate or heterogeneous concepts that can hinder development and collation of the evidence base. For example, there have been several recent funding calls related to the use of big data in public health research, including obesity [12-14]. One project, 'Big O', that was awarded funding under the Horizon 2020 Big Data funding call uses mobile phones to purposively collect data on obesity-related behaviours such as food intake [15]. While these data were deemed to meet the definition of big data in this instance, purposively collected data is a grey area, which is not always considered to constitute big data [5]. A definition of what constitutes big data would provide clear guidance and reduce inefficiencies for funders and researchers in understanding which proposed projects meet the funding criteria. It could also facilitate the use of particular datasets, and similar exposure and outcome variables which are imperative to enable meta-analyses and systematic reviews to summarise scientific evidence [16].

Developing a clear definition of big data for obesity research could also aid consistency and transparency across contributors from different industries and settings. One potential pitfall to progress in this field is the management and sharing of data [3, 4]. The General Data Protection Regulation (GDPR) [17] recently introduced across Europe provides a clear example of where not having a definition of big data for obesity research could be problematic. These new data regulations are accompanied by the threat of fines of up to 10 million Euros or $2 \%$ of global turnover for any personal data breaches including those related to collecting, processing or sharing data [18]. For egregious breaches, fines of 20 million Euro or $4 \%$ of global turnover are proposed. These substantial fines offer significant reason to hinder data collectors, particularly commercial companies, from sharing their data with researchers despite the potential for public benefit. Exemptions to the data regulations do exist for purposes in the public interest or for research. However, these terms have not been explicitly defined and appropriate safeguards relating to storage, processing and sharing are still required to protect anonymity [17]. Having a clear definition of big data for obesity research that could be adopted by member states may help to specify cases where exemptions to the regulations are appropriate.

Previous literature regarding big data has focused on analysis techniques or terminology [3, 9]. It has largely overlooked the practical elements necessary to guide successful acquisition and appropriate utilisation of big data for non-communicable conditions such as obesity [7, 19]. Thus in addition to a clear definition, there is need for an architecture for utilising big data in obesity research. This will help facilitate consistent and effective approaches and help overcome any issues academics may encounter. Previous authors exploring the usage of big data in health and social care have highlighted a number of challenges including data acquisition restrictions or costs that limit universal accessibility of datasets [4, 7]. Ethical and legal questions also exist around ownership and access, such as whether commercial data should be made available to research institutions for potential societal benefit [20]. Further, adherence to ethical principles and data protection regulations is problematic when individuals have not explicitly consented for their data to be used or linked to other data [21]. Additional challenges also include the need for data management and analysis skills that lie outside traditional public health training [22]. Furthermore, there are questions around data governance and reporting requirements as increasing numbers of people become involved in data creation and collation [23]. Similar concerns persist regarding bias, which can be introduced through poor data or study design quality, and in turn limit the ability to draw casual inference [24].

In recognition of the issues surrounding the use of big data, the Economic and Social Research Council (ESRC) funded a Strategic Network for Obesity (Obesity Network) 


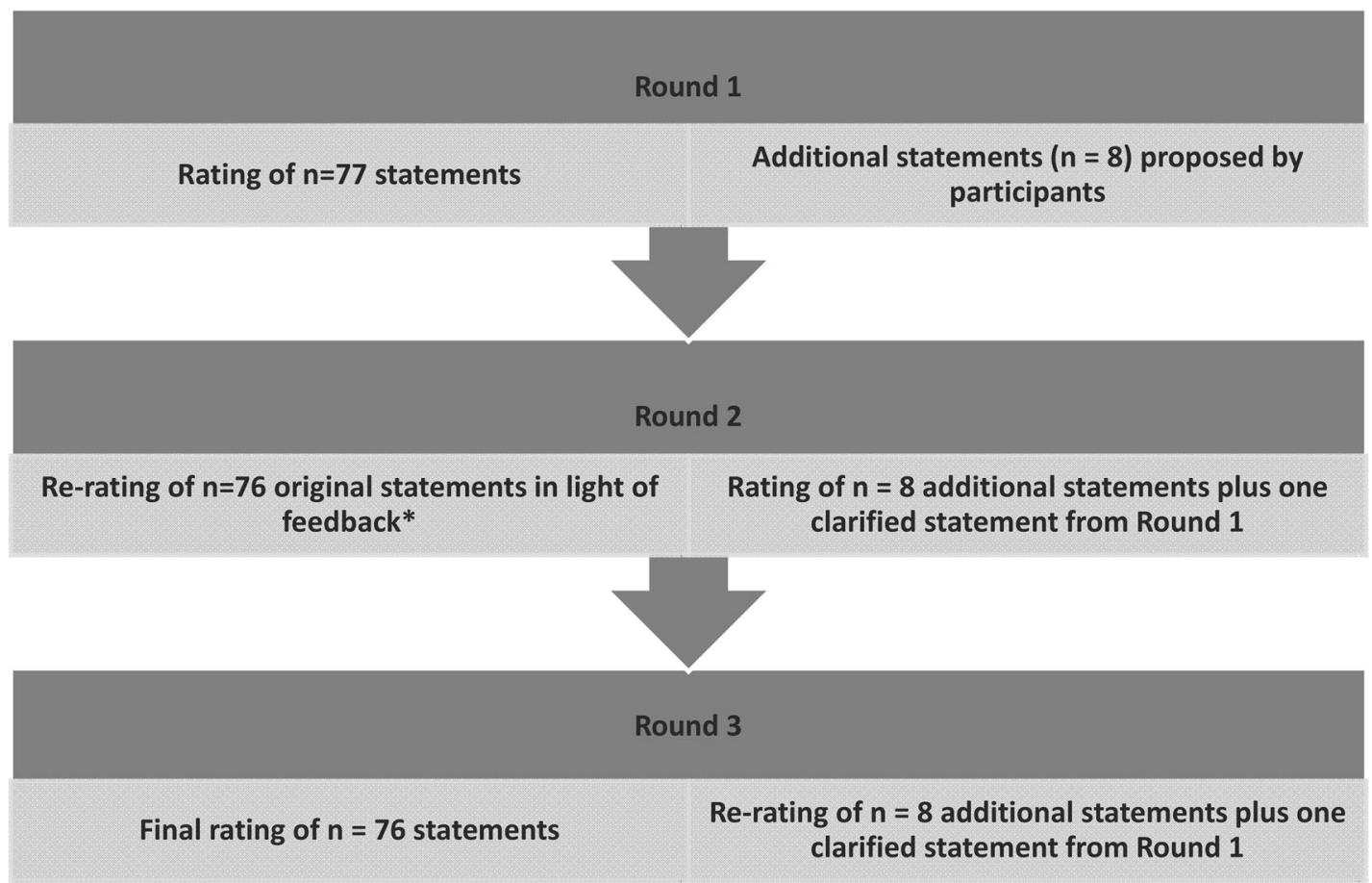

Fig. 1 Flow diagram illustrating the three survey rounds of the Delphi study. *One statement that appeared in Round 1 was removed, and a new clarified version was added in Round 2

[25]. This network is a collaboration of 40 members from academia, industry, health charities and the public sector that explored emerging forms of data to catalyse an approach to obesity at five network meetings between 2015 and 2017. These meetings highlighted that challenges to the effective application of big data to obesity research are experienced similarly across obesity-related disciplines by members of the Organisation for Economic Co-operation and Development (OECD). While there may be some minor differences in their use to account for local or cultural issues, the acquisition and employment of big data should be transferable between countries to enable international comparison. Thus the aim of the present study was to establish an agreed approach for using big data in obesityrelated research in OECD countries. A Delphi survey design was used to integrate international and interdisciplinary perspectives from academics with expertise in obesity research and/or experience applying big data to examine obesity, dietary or physical activity outcomes.

The objectives of this study were to build consensus among international experts in the field of obesity on: (i) a definition of big data that is appropriate for obesity research; and (ii) consistent and effective approaches academic researchers can take to use big data to address obesity with particular consideration of the issues relating to: Data Acquisition, Ethics, Governance, Training and Infrastructure, Reporting and Transparency, and Quality and Inference.

\section{Methods}

\section{Study design}

The Delphi technique has proven to be a reliable measurement instrument in developing new concepts and setting the direction of future-orientated research [26]. The technique seeks the opinion of a group of experts in order to assess the extent of agreement and to resolve disagreement on an issue [27]. It has been used to establish consensus across a range of subject areas, with several in the field of obesity and obesity-related behaviours [28-31].

The Delphi process comprised three rounds (Fig. 1). In Round 1, participants were asked to independently rank a total of 77 statements, across seven domains, using a 4point Likert scale ('strongly agree', 'agree', 'disagree', 'strongly disagree'). It has been demonstrated that 4-point scales produce stable findings in Delphi studies [32]. For each statement, participants were given the option to select 'don't know' as an alternative response. This option was added because big data is an emerging and challenging field, and feedback from pilot testing indicated that some participants may not know how to answer certain statements. Furthermore, this enabled identification of domains that are particularly unclear and require additional attention. A free-text response was available to participants within each of the survey domains, providing the opportunity to elaborate or explain responses. In Round 1, data on 
participant demographics were also collected including: gender, year of birth, country of residence, current job position, highest educational qualification obtained and time (in years) working in the field of obesity research.

In Round 2, each participant received an individualised survey comprising 85 statements, across seven domains. This survey included 76 statements from Round 1, which were presented alongside participants' own responses from Round 1, as well as the group's collective response (percentage agreement/disagreement) to each statement. All 'don't know' responses were excluded from the group response. Participants were asked to reconsider their responses in light of the group's responses. Round 2 also included eight new statements derived from the free-text responses to Round 1. Further, the free-text responses from Round 1 helped to clarify one statement which was then added as a new statement in Round 2. There was no option for free-text responses in Round 2.

In Round 3, each participant received an individualised survey, comprising all 85 statements from Round 2 presented alongside the participants' own responses and the group's response (percentage agreement/disagreement) from Round 2. Participants were asked to reconsider their responses in light of the group's responses for a final time.

Three survey rounds were employed because this enables adequate reflection on group responses and is considered optimal to reach consensus [33]. Three survey rounds also allowed free-text responses from Round 1 to be incorporated as new statements in Round 2 and re-evaluated in light of the group consensus in Round 3 (Fig. 1). A third survey round for these new statements was not required because consensus was achieved on all except one statement which had response split that meant consensus would be unlikely. All surveys were administered using Qualtrics (Provo, USA), and survey links were distributed via email.

\section{Survey development}

Statements for the survey were developed from study team's expertise, intelligence from the Obesity Network and a review of the literature [5]. To meet the study objectives, the survey was divided into two sections. The first included statements to establish a definition of big data and the second, sought consensus on approaches to using big data in obesity research. Statement development capitalised on an existing survey carried out as part of Obesity Network activities. Members' responses to the question: 'what is big data?' were used to create statements for the first section of the survey; and the second section used members' responses to the questions: "what are your concerns with using big data for research?' and 'what are the main challenges within your work in terms of big data?' Four authors (SZ, CG, MH and $\mathrm{EW}$ ) independently analysed the responses to identify themes and propose statements. These statements were supplemented and refined in light of the literature review findings and knowledge of the research team.

A total of 14 statements were included in the first section of the survey. The second section included 63 statements across six domains: Data Acquisition, Ethics, Governance, Training and Infrastructure, Reporting and Transparency, and Quality and Inference. These domains have also been identified as important considerations surrounding the use of big data in health and social care [7, 20-24]. The survey statements were constructed to highlight the key challenges and opportunities relating to each domain, and to agree effective approaches to address these challenges.

The survey was piloted with seven academics who had a range of obesity-related experience, including professors in statistical epidemiology, nutritional science and behavioural science. An iterative processes of feedback was undertaken to improve the structure and readability of statements, and to determine whether any additional statements were needed.

\section{Expert panel recruitment}

In Delphi exercises, a minimum of 12 respondents is generally considered to be sufficient to enable consensus to be achieved, larger sample sizes can provide diminishing returns regarding the validity of the findings [34-39]. Nevertheless, Delphi sample sizes depend more on group dynamics in reaching consensus than their statistical power [40, 41]. A non-probability purposive sample of ninety-six participants were invited via email to participate in this Delphi survey. Sampling was purposive to ensure that invited participants met the inclusion criteria. All participants were required to be 18 years or above, fluent English speakers, actively conducting research in obesity or obesityrelated fields, and affiliated with an academic institution from an OECD country. The invited participants were either members of the Obesity Network $(n=34)$, academics known to members of the Obesity Network $(n=45)$, or authors of published articles relating to obesity and big data identified from the first paper in this series [5] $(n=17)$. To complete the Delphi process, participants were required to respond across all three rounds. Therefore, those who did not respond to Round 2 were not invited to participate in Round 3. A dropout rate of $20 \%$ was expected over the three rounds, in accordance with previous Delphi studies $[32,42]$. This study aimed to recruit and complete the process with 30 experts.

\section{Ethics}

Ethical approval for this study was granted by the Local Research Ethics Committee at Leeds Beckett University. 
All participants provided informed consent to take part at the beginning of the process as part of the online survey. All data were handled in accordance with UK data protection regulations.

\section{Data analysis}

Descriptive statistics were used to describe participants' demographic characteristics and group responses to each statement in all three rounds. Consensus was defined as > $70 \%$ of participants agreeing/strongly agreeing or disagreeing/strongly disagreeing with a statement in Round 3. This level of agreement has been considered appropriate in previous Delphi studies [40, 42, 43]. All 'don't know' responses were excluded from the group response to ensure that the reported percentage agreement or disagreement for each statement represented the consensus among only those who felt they knew the answer. Stability of consensus was considered reached if the between round group responses varied by $\leq 10 \%$ [44]. Analyses were conducted using SPSS for windows version 24 [45].

\section{Results}

Of the 96 experts invited to participate in this Delphi study, 36 participants completed Round 1 (37.5\% response rate), 29 of 36 completed Round 2 (80.6\% response rate) and 26 of 29 completed Round 3 ( $89.7 \%$ response rate). Table 1 presents the demographic characteristics of participants in each round. Gender distribution was consistent across the three rounds, with only a slightly higher percentage of males. Participants' mean age ranged from 42 to 44 years across the three rounds, and approximately three quarters resided in the UK. The majority of respondents were senior academics, had doctoral degrees and had been working in the field of obesity research for $\geq 5$ years.

Table 2 shows a summary of the Delphi statements for each of the seven domains. The number of statements where consensus was achieved improved for each domain from Round 1 to Round 3. In Round 1, consensus was achieved for $64.5 \%(n=49)$ of the 76 statements. In Round 2, consensus was achieved for $81.2 \%(n=69)$ of the 85 statements and this rose to $90.6 \%(n=77)$ in Round 3. There was variation in the proportion of statements that achieved consensus between domains but the proportion of consensus increased in each subsequent round across all domains. By Round 3, 100\% consensus was achieved for three domains (Definition of Big Data $(n=15)$, Data Governance $(n=5)$, and Quality and Inference $(n=11)$; the lowest level of consensus was $75.0 \%$ for Training and Infrastructure $(n=9)$. Stability of consensus $(<10 \%$ variation) was achieved between Round 2 and Round 3 for four of the seven domains.
Table 1 Demographic characteristics of Delphi participants

\begin{tabular}{|c|c|c|c|}
\hline & $\begin{array}{l}\text { Round } 1 \\
(n=36)\end{array}$ & $\begin{array}{l}\text { Round } 2 \\
(n=29)\end{array}$ & $\begin{array}{l}\text { Round } 3 \\
(n=26)\end{array}$ \\
\hline \multicolumn{4}{|l|}{ Gender } \\
\hline Male & $52.8 \%$ & $55.2 \%$ & $53.8 \%$ \\
\hline Female & $47.2 \%$ & $44.8 \%$ & $46.2 \%$ \\
\hline $\begin{array}{l}\text { Mean age in years } \\
\text { (SD) }\end{array}$ & $43.9(10.9)$ & $42.3(9.8)$ & $41.5(9.7)$ \\
\hline \multicolumn{4}{|l|}{$\begin{array}{l}\text { Country of } \\
\text { residence }\end{array}$} \\
\hline UK & $72.2 \%$ & $75.9 \%$ & $80.8 \%$ \\
\hline USA & $11.1 \%$ & $6.9 \%$ & $7.7 \%$ \\
\hline Netherlands & $8.3 \%$ & $10.3 \%$ & $7.7 \%$ \\
\hline New Zealand & $2.8 \%$ & $3.4 \%$ & $3.8 \%$ \\
\hline Australia & $2.8 \%$ & $3.4 \%$ & $0.0 \%$ \\
\hline Ireland & $2.8 \%$ & $0.0 \%$ & $0.0 \%$ \\
\hline \multicolumn{4}{|l|}{ Current role } \\
\hline Professor & $30.6 \%$ & $27.6 \%$ & $26.9 \%$ \\
\hline $\begin{array}{l}\text { Associate } \\
\text { Professor }\end{array}$ & $13.9 \%$ & $13.8 \%$ & $15.4 \%$ \\
\hline Lecturer & $16.7 \%$ & $17.2 \%$ & $15.4 \%$ \\
\hline $\begin{array}{l}\text { Research } \\
\text { Fellow }\end{array}$ & $27.8 \%$ & $31.0 \%$ & $30.8 \%$ \\
\hline PhD student & $5.6 \%$ & $6.9 \%$ & $7.7 \%$ \\
\hline Other & $5.6 \%$ & $3.4 \%$ & $3.8 \%$ \\
\hline \multicolumn{4}{|l|}{ Education } \\
\hline $\begin{array}{l}\text { Doctoral } \\
\text { Degree }\end{array}$ & $91.7 \%$ & $93.1 \%$ & $92.3 \%$ \\
\hline $\begin{array}{l}\text { Professional } \\
\text { Fellow }\end{array}$ & $2.8 \%$ & $0.0 \%$ & $0.0 \%$ \\
\hline $\begin{array}{l}\text { Master's } \\
\text { Degree }\end{array}$ & $5.6 \%$ & $6.9 \%$ & $7.7 \%$ \\
\hline \multicolumn{4}{|l|}{$\begin{array}{l}\text { Years working in } \\
\text { the field }\end{array}$} \\
\hline $10+$ years & $52.8 \%$ & $44.8 \%$ & $46.2 \%$ \\
\hline $6-9$ years & $14.0 \%$ & $17.2 \%$ & $15.3 \%$ \\
\hline $4-5$ years & $19.4 \%$ & $20.7 \%$ & $19.2 \%$ \\
\hline $1-3$ years & $13.9 \%$ & $17.2 \%$ & $19.2 \%$ \\
\hline
\end{tabular}

Table 3 presents the group responses to each survey statement included in the definition of Big Data domain. By Round 3, consensus was achieved for all 15 statements, with $80 \%(n=12)$ of these statements achieving consensus in Round 2. Three statements needed three rounds before consensus was reached. Table 4 shows the group responses to the Delphi statements as they appeared in the participant survey across the six domains. The Delphi survey sought to identify agreed approaches to using big data in obesity research. For the Data Acquisition domain, eleven (68.8\%) of the 16 statements reached consensus in Round 2; by Round 3 consensus has been achieved on 13 statements (81.3\%). Three statements did not reach consensus and these related to 
Table 2 Summary of grouped statements by domain

\begin{tabular}{|c|c|c|c|c|c|c|}
\hline \multirow[t]{2}{*}{ Statement domains } & \multicolumn{3}{|c|}{$\begin{array}{l}\text { Number of statements in each } \\
\text { domain }\end{array}$} & \multicolumn{3}{|c|}{$\begin{array}{l}\text { Proportion of statements where consensus } \\
\text { was achieved }(n)\end{array}$} \\
\hline & Round 1 & Round 2 & Round 3 & Round 1 & Round 2 & Round 3 \\
\hline Definition of Big Data & 14 & 15 & 15 & $64.3 \%(9)$ & $80.0 \%(12)$ & $100.0 \%(15)$ \\
\hline Data Acquisition & $13^{\mathrm{a}}$ & 16 & 16 & $38.5 \%(5)$ & $68.8 \%(11)$ & $81.3 \%(13)$ \\
\hline Ethics & 13 & 15 & 15 & $61.5 \%(8)$ & $80.0 \%(12)$ & $93.3 \%(14)$ \\
\hline Data Governance $^{\mathrm{b}}$ & $5^{\mathrm{a}}$ & 5 & 5 & $80.0 \%$ (4) & $100.0 \%(5)$ & $100.0 \%(5)$ \\
\hline Training and Infrastructure ${ }^{\mathrm{b}}$ & 11 & 12 & 12 & $63.6 \%(7)$ & $75.0 \%$ (9) & $75.0 \%$ (9) \\
\hline Reporting and Transparency ${ }^{\mathrm{b}}$ & 9 & 11 & 11 & $77.8 \%(7)$ & $90.9 \%(10)$ & $90.9 \%(10)$ \\
\hline Quality and Inference $^{\mathrm{b}}$ & 11 & 11 & 11 & $81.8 \%(9)$ & $90.9 \%(10)$ & $100.0 \%$ \\
\hline Totals & 76 & 85 & 85 & $64.5 \%$ (49) & $81.2 \%(69)$ & $90.6 \%$ (77) \\
\hline
\end{tabular}

Note: Consensus was achieved when $70 \%$ of participants strongly agreed/agreed or strongly disagreed/ disagreed with a statement

a Statements in this round of this domain include responses where 'don't know' exceeded $30 \%$ of total responses

${ }^{\mathrm{b}}$ Stability of consensus ( $<10 \%$ variation) was achieved between Round 2 and Round 3 participant knowledge, big data owners' responsibilities for promoting their data, and data protection regulations. For the Ethics domain, $14(93.3 \%)$ of the 15 statements reached consensus by Round 3, up from $12(80.0 \%)$ in Round 2. One statement relating to the ethics of commercial companies withholding big datasets could not be agreed upon by the group. Consensus was achieved for all five (100\%) statements included in the Data Governance domain in Rounds 2 and 3. In Round 1, however, more than $30 \%$ of participants reported not knowing whether data governance processes were clear for data owners and controllers. For the Training and Infrastructure domain, consensus was reached for 9 $(75.0 \%)$ of the 12 statements in Rounds 2 and 3. No consensus was achieved for three statements, highlighting differences in time, training and equipment needs for big data analyses across researchers and institutions. One statement out of 11 in the Reporting and Transparency domain could not be agreed by the expert panel; this statement described the need to report costs associated with acquiring big data. The remaining 10 (90.9\%) statements achieved consensus in Round 2 and Round 3. Consensus was attained for all 11 (100.0\%) statements included in the Quality and Inference domain by Round 3, an improvement from 9 (81.8\%) in Round 1 and 10 (90.9\%) in Round 2. Across all domains, the direction of agreement for statements not reaching consensus until the third round did not change from the earlier rounds, it only strengthened.

The proportion of participants that reported 'don't know' to each statement in Round 3 is presented in Table S1, Supplementary material. The Definition of Big Data domain had the lowest proportion of 'don't know' responses (1.5\%), and the Data Governance domain had the highest $(12.3 \%)$. None of the statements in Round 3 had 'don't know' responses that exceeded $30 \%$ of the total responses.

\section{Discussion}

This Delphi survey achieved consensus, from a panel of 26 international experts who completed three rounds, on $100 \%$ of the 15 statements proposed to develop a definition of big data for obesity research. Additionally, the survey reached consensus on $88.6 \%$ of statements put forward to describe approaches for researchers to effectively use big data in obesity-related studies. Descriptions of the panel agreement against the two aims of this study are outlined below under the subheadings 'defining big data' and 'consistent approaches to using big data'.

\section{Defining big data}

One definition that represents the consensus among the expert group on the full list of definition-specific descriptors is provided in the box below. This type of definition is likely to be important when communicating what big data is to those not familiar with the term or when ascertaining the circumstances in which big data applies to, or is exempt from, regulations. For audiences more familiar with big data, a shorter, more succinct definition may be more appropriate.

Big data is always digital, has a large sample size, and a large volume or variety or velocity of variables that require additional computing power. It can include quantitative, qualitative, observational or interventional data from a wide range of sources (e.g. government, commercial, cohorts) that have been collected for research or other purposes, and may include one or several datasets. Specialist skills in computer programming, database management and data science analytics are usually required to analyse big data. 
Table 3 Responses to statements included in the Definition of Big Data domain

\begin{tabular}{|c|c|c|c|c|c|c|}
\hline \multirow[t]{2}{*}{ Big Data.... } & \multicolumn{2}{|c|}{ Round $1(n=36)$} & \multicolumn{2}{|c|}{ Round $2(n=29)$} & \multicolumn{2}{|c|}{ Round $3(n=26)$} \\
\hline & Agree $\%$ & Disagree $\%$ & Agree $\%$ & Disagree $\%$ & Agree $\%$ & Disagree $\%$ \\
\hline 1. Always has a large sample size & $\mathbf{7 7 . 8 \%}$ & $22.2 \%$ & $\mathbf{7 5 . 9 \%}$ & $24.1 \%$ & $\mathbf{8 8 . 5 \%}$ & $11.5 \%$ \\
\hline 2. Always requires additional computing power & $68.6 \%$ & $31.4 \%$ & $69.0 \%$ & $31.0 \%$ & $80.8 \%$ & $19.2 \%$ \\
\hline $\begin{array}{l}\text { 3. Is never collected for research purposes (i.e. there is no a priori } \\
\text { research question) }\end{array}$ & $25.7 \%$ & $74.3 \%$ & $21.4 \%$ & $78.6 \%$ & $15.4 \%$ & $84.6 \%$ \\
\hline 4. Is always observational & $40.0 \%$ & $60.0 \%$ & $22.2 \%$ & $\mathbf{7 7 . 8 \%}$ & $23.1 \%$ & $76.9 \%$ \\
\hline $\begin{array}{l}\text { 5. Does not require specialist mathematical or data science analytical } \\
\text { skills }\end{array}$ & $12.1 \%$ & $87.9 \%$ & $7.1 \%$ & $92.9 \%$ & $8.0 \%$ & $92.0 \%$ \\
\hline 6. Does not require specialist knowledge of database management & $15.6 \%$ & $84.4 \%$ & $14.8 \%$ & $85.2 \%$ & $12.5 \%$ & $87.5 \%$ \\
\hline 7. Does not require knowledge of computer programming & $42.4 \%$ & $57.6 \%$ & $37.0 \%$ & $63.0 \%$ & $25.0 \%$ & $\mathbf{7 5 . 0 \%}$ \\
\hline 8. Is always digital & $61.8 \%$ & $38.2 \%$ & $66.7 \%$ & $33.3 \%$ & $72.0 \%$ & $28.0 \%$ \\
\hline 9. Does not include qualitative data & $35.3 \%$ & $64.7 \%$ & $17.9 \%$ & $82.1 \%$ & $23.1 \%$ & $76.9 \%$ \\
\hline 10. Includes government data sets & $94.3 \%$ & $5.7 \%$ & $96.6 \%$ & $3.4 \%$ & $96.2 \%$ & $3.8 \%$ \\
\hline 11. Includes cohort data sets & $86.1 \%$ & $13.9 \%$ & $92.9 \%$ & $7.1 \%$ & $96.2 \%$ & $3.8 \%$ \\
\hline 12. Includes commercial data sets & $97.2 \%$ & $2.8 \%$ & $96.6 \%$ & $3.4 \%$ & $96.2 \%$ & $3.8 \%$ \\
\hline 13. Includes routine data sets & $94.4 \%$ & $5.6 \%$ & $96.6 \%$ & $3.4 \%$ & $100.0 \%$ & $0.0 \%$ \\
\hline 14. Always includes more than one data set & $16.7 \%$ & $83.3 \%$ & $17.2 \%$ & $82.8 \%$ & $15.4 \%$ & $84.6 \%$ \\
\hline $\begin{array}{l}\text { 15. Big data always has at least one of: } \\
\text { - large volume (e.g. in terms of sample size, number of variables or } \\
\text { measurement occasions), } \\
\text { - variety (e.g. in terms of the types of variable), or } \\
\text { - velocity (e.g. is generated at speed) }\end{array}$ & - & - & $93.1 \%$ & $6.9 \%$ & $92.3 \%$ & $7.7 \%$ \\
\hline
\end{tabular}

Note: Bold \% denotes that $70 \%$ consensus was achieved

This definition of big data determined by Delphi method draws upon the increasingly recognised definition of the three V's of big data: volume, variety and velocity [2-4]. However, it provides greater detail about types of information equated with the term and the sources from which it can be acquired. It also recognises that training and computing resources required for big data extend beyond those traditionally used in obesity studies. This definition is consistent with descriptions provided in commentaries by authors from North America with regard to big data use in epidemiological or public health research [7, 24], providing confidence in the representativeness of our findings. The high level of agreement from this study's expert group in how we define big data for obesity research supports the notion that big data's defining characteristics are applicable across countries and in different research contexts.

Given the evolving nature of big data in obesity research in many countries, the key descriptors agreed upon can be employed in versatile ways. For example, peer-reviewed journals could require authors to follow a reporting protocol, such as BEE-COAST [6], when describing their big data studies and may define such studies using one or more of the definition descriptors agreed upon by the expert panel. These may include data type (e.g. requiring data to be digital with a large sample size, volume, variety and/or velocity) and source descriptors (e.g. requiring data to be from government, commercial or cohort sources) but could exclude the training descriptors.

\section{Consistent approaches to using big data}

The consensus-building technique employed in this study identified a number of approaches that need to be consistently implemented by various stakeholders to optimise use of big data in obesity research. Figure 2 summarises six challenges the expert panel collectively identified as currently hindering effective use of big data and the recommended six different stakeholders groups who are optimally placed to become agents of change to overcome these challenges. Informed by the consensus achieved on 62 statements, the figure also illustrates the potential solutions the expert panel agreed could be enacted by each stakeholder group to facilitate effective and consistent use of big data in obesity-related research.

The results of this study identified a number of issues surrounding big data that have been previously noted, including disparities in acquisition due to cost, access and time constraints [4, 7], and ethical concerns regarding individual and commercial privacy and consent [3, 20, 23]. This Delphi study, however, expanded on previous 


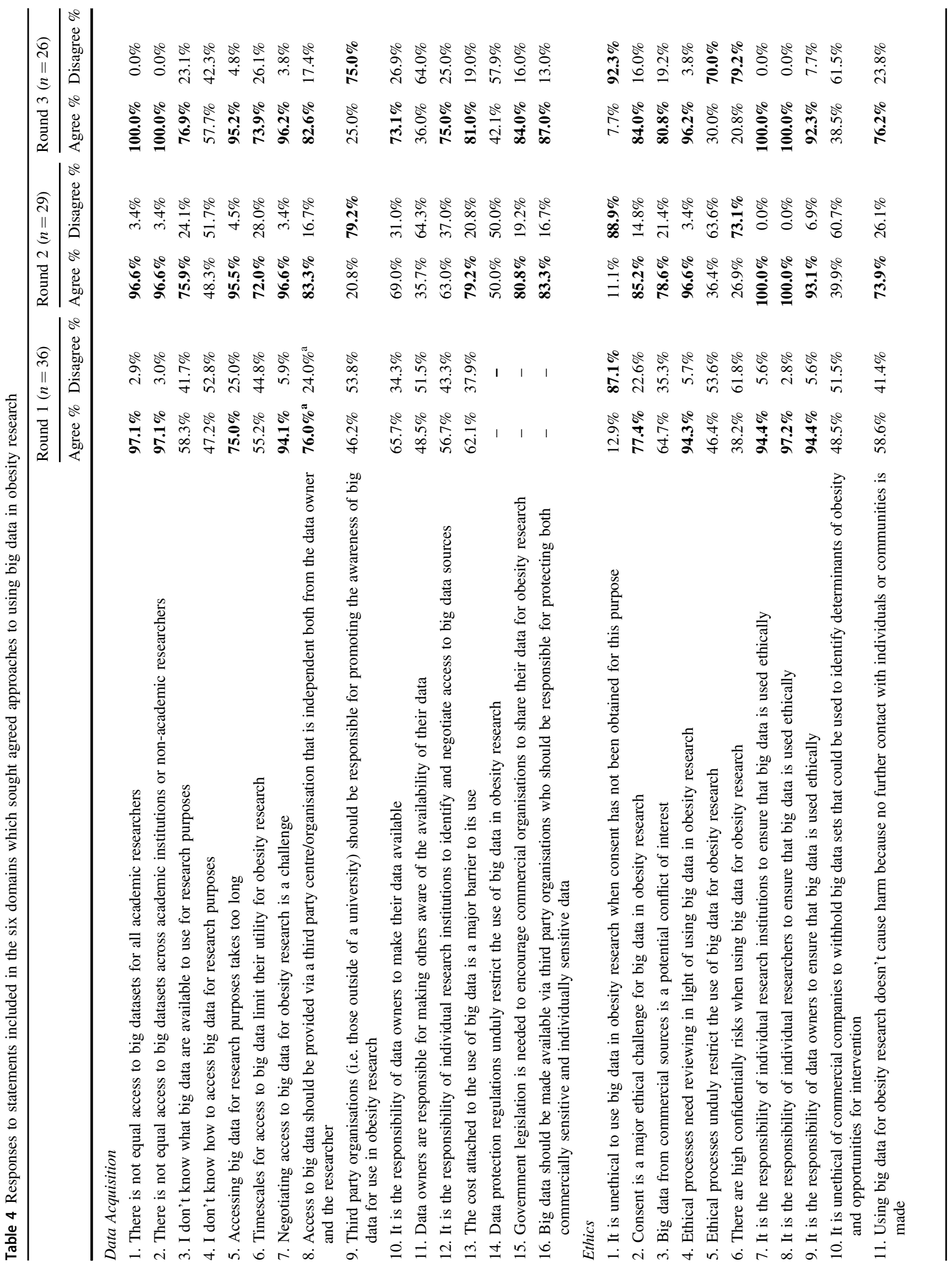




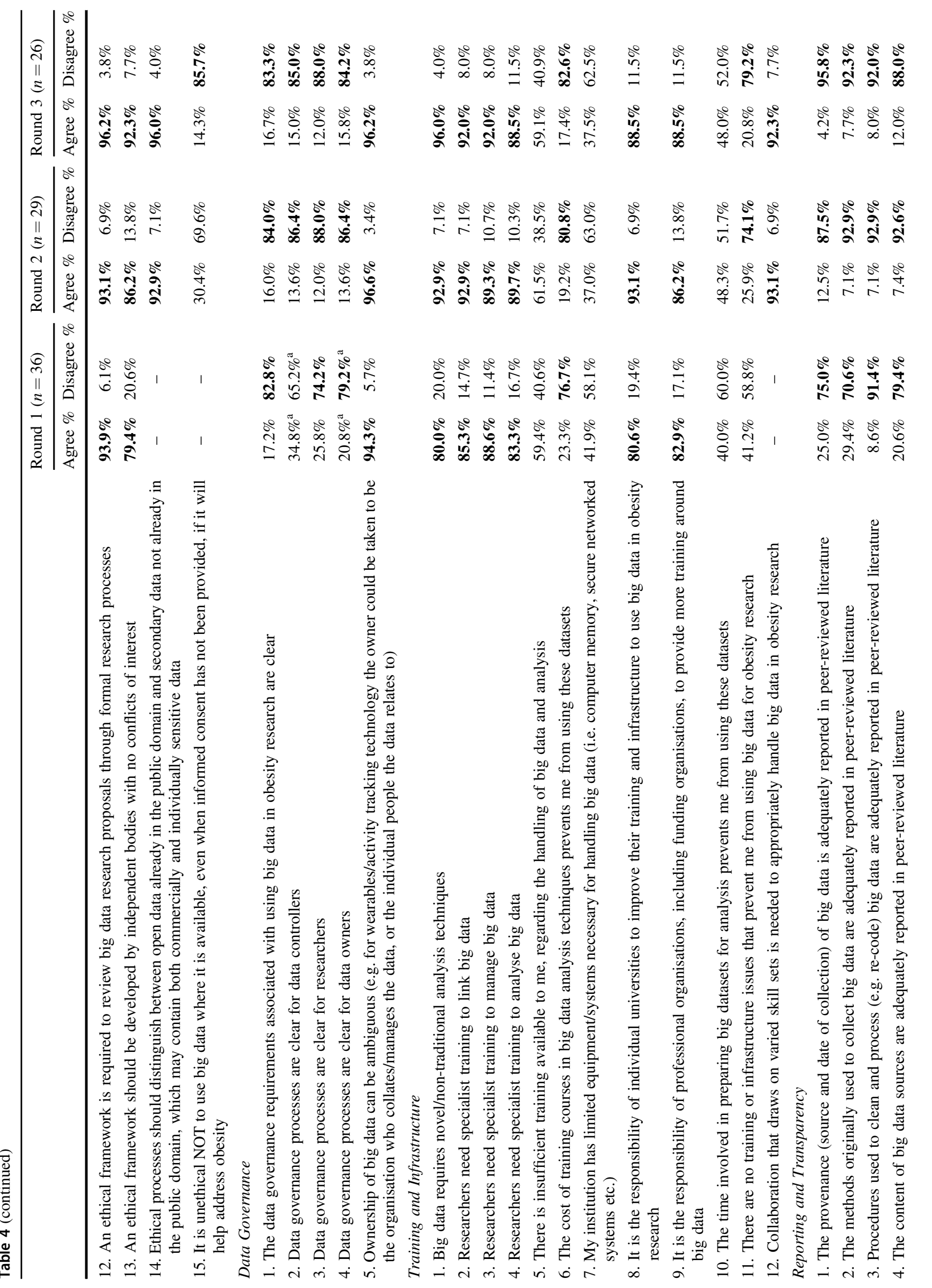




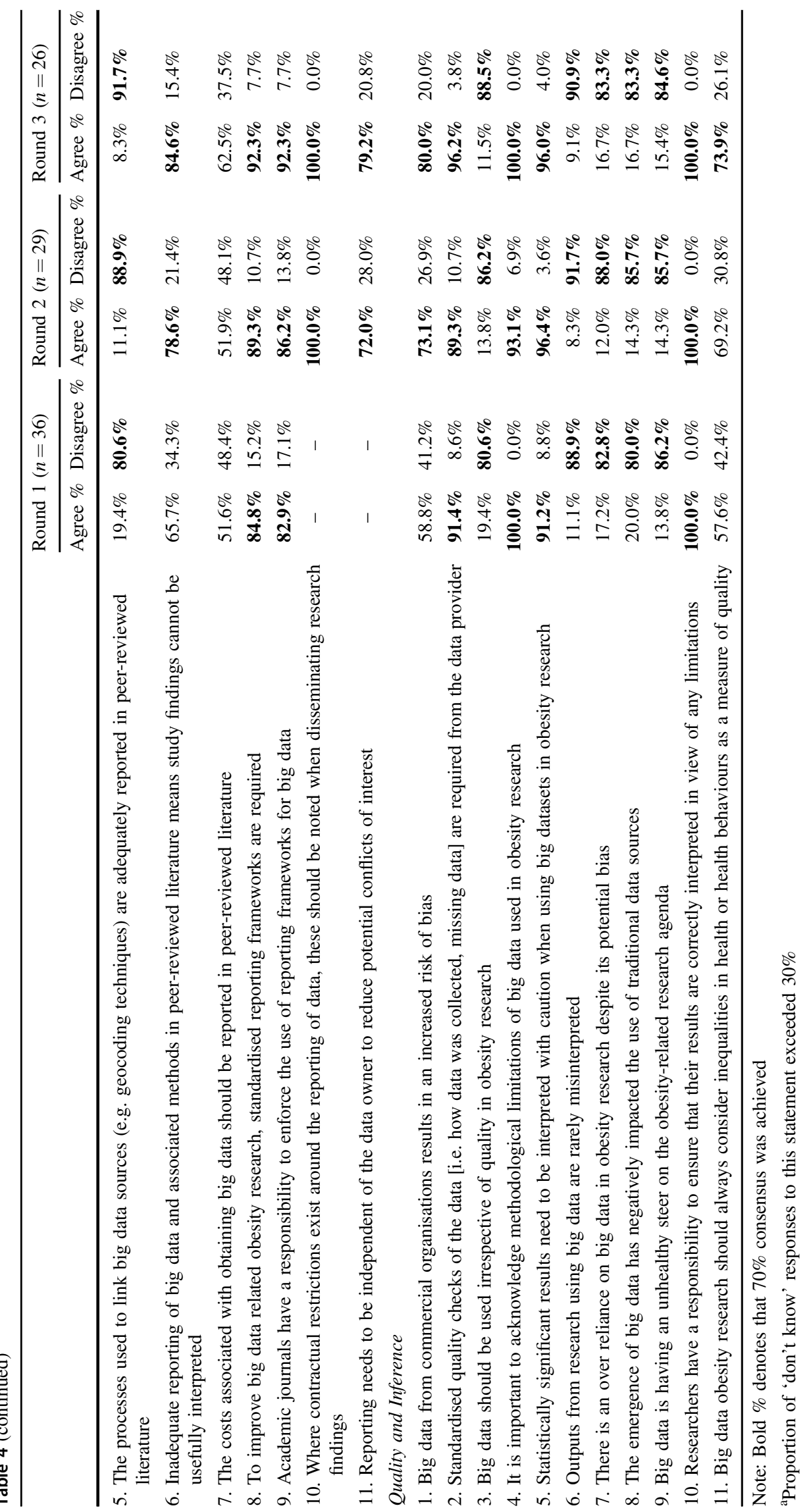




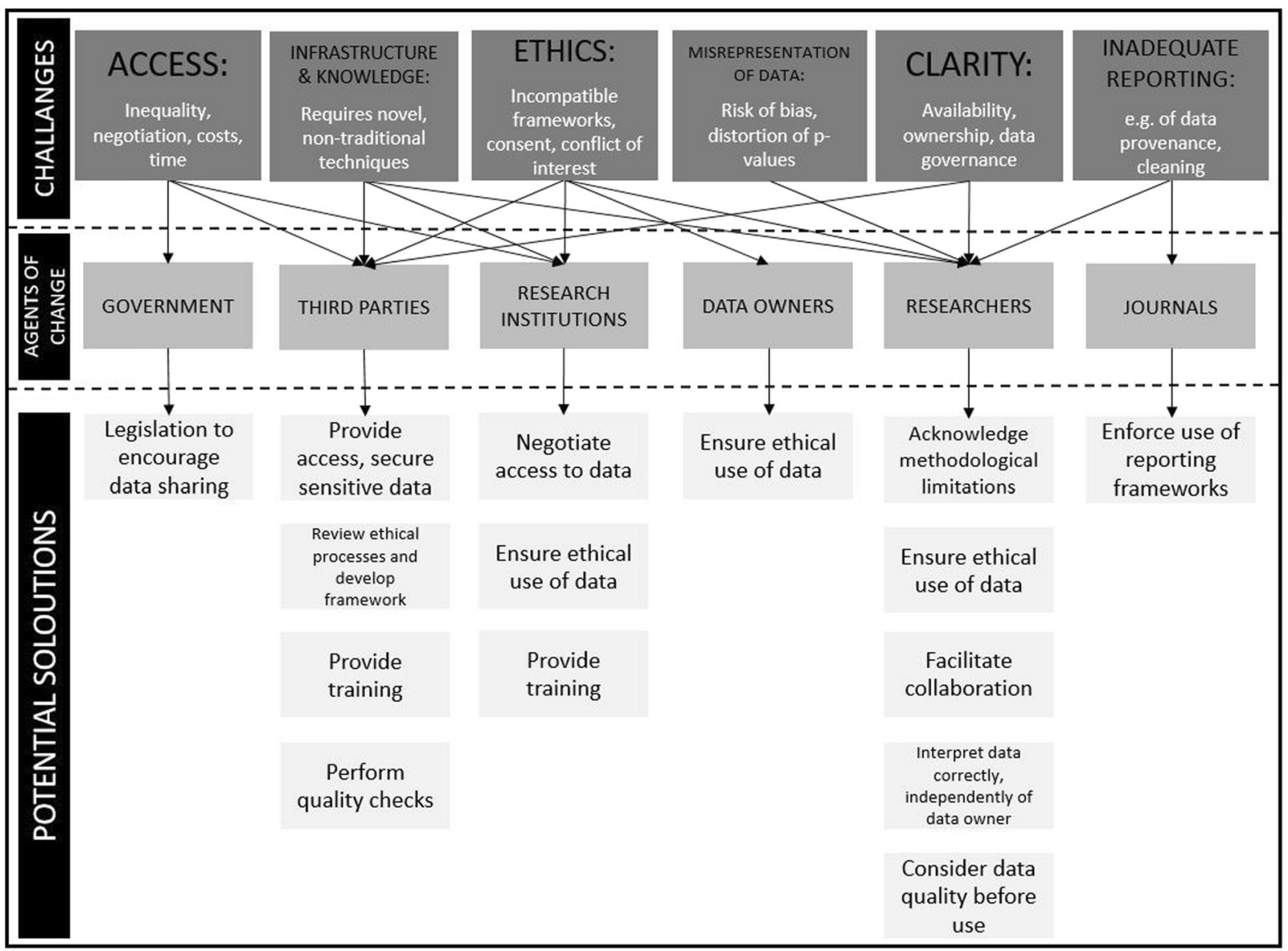

Fig. 2 Challenges, solutions and agents of change for effective use of big data in obesity research

literature by identifying practical actions to overcome these challenges. A recurring theme was around the need for third party action. For example, the experts agreed that there was a need for organisations that act independently of both data owners and researchers, to provide repositories of big datasets from various sources and ensure the protection of both individual identities and commercial sensitivities. A small number of such organisations already exist, including the Consumer Data Research Centre (CDRC) and Administrative Data Research Centre (ADRC) that form part of the ESRC-funded Administrative Data Research Network and are hosted by UK universtities [46, 47]. These centres provide access to a variety of data sources for the research community, potentially reducing time and financial costs.

Such third parties work with commercial and government organisations to encourage them to open up their data to researchers to help address societal issues like obesity. These repository centres could extend their current remit to include advocating for government legislation to require commercial organisations to share their data for obesity research as supported by the expert panel in this study. Such third party organisations can also safeguard commercially and individually sensitive data, by providing secure facilities for approved researchers to access linked, deidentified data. Techniques such as data perturbation can and are being used by third party repository centres to enable functionally anonymised data to be used in research [22].

The expert panel from this study also agreed that an ethical framework and data governance protocols need to be developed by a non-conflicted, independent body to guide appropriate use of big data by all stakeholders. Potential organisations could include the Obesity Network or internationally recognised professional organisations such as the World Obesity Federation or International Society for Behavioural Nutrition and Physical Activity. The results of this study provide useful information to draft ethical and governance protocols that could then be debated and subsequently agreed at international conferences. Specifying the requirements for different actors, including data owners, data controllers and researchers, will aid adherence to high ethical and data governance standards. Endorsement of the protocols by a range of professional and government organisations would facilitate their uptake and implementation by academic ethical committees, third party data repositories, researchers and data owners. 
It has become increasingly recognised that the growth of big data requires specific analytic skills that are not traditionally incorporated into professional training courses for a number of sectors including public health and epidemiology $[2,9,22,48]$. When considering training needs, panellists from this study recommended that universities, professional organisations and funding bodies provide more teaching in linking, managing and analysing big data. Such training opportunities could take the form of continuing professional development activities or incorporated into undergraduate and postgraduate curriculums. For example, professional organisations such as the World Obesity Federation could introduce training opportunities in machine learning techniques $[7,9,49]$ as part of their E-learning modules. Data repository centres, including CDRC and ADRC mentioned above, also offer a range of short courses about big data linkage, management and analysis that are currently available to researchers to improve their confidence and skills in this area. While a number of online training facilities are freely available, funding bodies may need to do more to support skill development in big data analytics for researchers at all career stages.

The application of machine learning techniques to big data in obesity research has been shown to provide robust methods for handling missing and incorrectly recorded data eliminating the need to curate longitudinal datasets for analyses [50]. However, concerns about data quality and causal inference with big data have been acknowledged [7, 21] and were supported by the expert panel in this study. Big data sources may not be representative, and similar data sources may not reveal consistent results. Additionally, while larger sample sizes reduce the likelihood of random error, measurement error can introduce bias independent of a dataset's sample size [3,51]. The experts participating in this study agreed that the methodological limitations of big data, including selection bias, measurement error and risk of confounding, should always be acknowledged. They indicated the need for standardised reporting frameworks to improve transparency regarding data quality and facilitate appropriate inference. The BEE-COAST framework [6] has been shown to suitably summarise the important features of a number of big data sources. If this framework were to be enforced by academic journals, and details outlining the background to data collection, data ownership, content and the temporality of the dataset routinely described, concerns about conflicts of interest and data quality are likely to be systematically reduced. The third parties proposed above could take an active role in promoting the adoption of this framework by editorial boards of peer-reviewed journals in a similar way to which reporting frameworks for observational studies and systematic reviews have been embraced $[52,53]$.

\section{Strengths and weaknesses}

This Delphi study gathered consensus on a range of topics relevant to the burgeoning global field of big data in academic obesity research, the findings for which have enabled the research team to develop initial guidance and areas for policy and research development. Drawing on an international network of obesity researchers funded to develop this field, views were gathered from a wide range of related disciplines. The size and composition of the expert panel may not be representative of all OECD countries and may therefore reduce the generalizability of the results. Nevertheless, one of the strengths of this paper is that the final sample size was more than double the lower limit threshold of 12 [39]. Given the global scale upon which this field operates, the Delphi consensus technique, which can be conducted online, was the appropriate tool for bringing together these views. In addition to identifying areas of consensus, the study was able to highlight areas where there is less certainty in the field, potentially requiring further exploration and a widening of disciplines to resolve these issues. While a strength of the study was its ability to access a network of colleagues in the field of obesity research, the authors of this study are members of the Obesity Network and this may have introduced some response bias. The response rates for each round of the study were $37.5 \%$, $80.6 \%$ and $89.7 \%$ for Round 1, Round 2 and Round 3, respectively. Based on guidance from the NIHR Health Technology Assessment for this technique [39], we anticipated a dropout rate of $20 \%$ over the three rounds of consensus development. A main limitation of this study is that it does not offer definitive guidance; however, this study recommends independent parties draw upon these findings and others to create resources to improve consistency and quality of big data use in the field of obesity.

\section{Conclusion}

With an expert panel, this study was able to reach consensus on the majority of statements included in this study. It was felt that the definition of big data in the context of obesity research was more nuanced than the simple and oft-cited three V's of big data: 'volume, variety and velocity', and includes quantitative, qualitative, observational or interventional data from a wide range of sources (e.g. government, commercial, cohorts) that have been collected for research or other purposes. This definition can help position future discussions and frameworks around the use of big data in obesity research.

Experts identified a number of challenges that need to be resolved in order to more effectively use big data in obesity research. A recurring theme was the need for third party 
action, for example to develop frameworks for reporting and ethics, to clarify data governance requirements and to support training and skill development. The findings also indicate that third parties should play a role in arbitrating access to big data in order to protect commercial and individual confidentiality, as well as enable more equitable access to data and potentially reduce the time and financial costs to individual researchers and institutions. While organisations that fulfil some of these roles already exist, further advocacy will likely be needed to encourage organisations to adopt wider responsibilities. Individual researchers, research institutions and data owners also hold important roles in facilitating effective and ethical use of big data. Determining the responsibilities of different actors, and monitoring adherence to these responsibilities will not be simple, and may require government involvement.

Acknowledgements The ESRC Strategic Network for Obesity was funded via Economic and Social Research Council grant number ES/ N00941X/1. The authors would like to thank all of the network investigators $^{\mathrm{a}}$ and members ${ }^{\mathrm{b}}$ for their participation in network meetings and discussion which contributed to the development of this paper. ${ }^{a}$ - www.cdrc.ac.uk/research/obesity/investigators/. ${ }^{b}$ - www. cdrc.ac.uk/research/obesity/network-members/

\section{Compliance with ethical standards}

Conflict of interest The authors declare that they have no conflicts of interest.

Publisher's note: Springer Nature remains neutral with regard to jurisdictional claims in published maps and institutional affiliations.

Open Access This article is licensed under a Creative Commons Attribution 4.0 International License, which permits use, sharing, adaptation, distribution and reproduction in any medium or format, as long as you give appropriate credit to the original author(s) and the source, provide a link to the Creative Commons license, and indicate if changes were made. The images or other third party material in this article are included in the article's Creative Commons license, unless indicated otherwise in a credit line to the material. If material is not included in the article's Creative Commons license and your intended use is not permitted by statutory regulation or exceeds the permitted use, you will need to obtain permission directly from the copyright holder. To view a copy of this license, visit http://creativecommons. org/licenses/by/4.0/.

\section{References}

1. Abarca-Gómez L, Abdeen ZA, Hamid ZA, Abu-Rmeileh NM, Acosta-Cazares B, Acuin C, et al. Worldwide trends in body-mass index, underweight, overweight, and obesity from 1975 to 2016: a pooled analysis of 2416 population-based measurement studies in 128.9 million children, adolescents, and adults. Lancet. 2017;390:2627-42.

2. McAfee A, Brynjolfsson E. STRATEGY \& COMPETITION Big Data: the management revolution. Harv Bus Rev. 2012;90:60-+.

3. Kaisler S, Armour F, Espinosa JA, Money W. Big Data: issues and challenges moving forward. 46th Hawaii International Conference on System Sciences. IEEE, 2013. p. 995-1004.
4. Mittelstadt BD, Floridi L. The Ethics of Big Data: Current and Foreseeable Issues in Biomedical Contexts. Sci Eng Ethics. 2016;22:303-41.

5. Timmins KA, Green MA, Radley D, Morris MA, Pearce J. How has big data contributed to obesity research? A review of the literature. Int J Obes (Lond). 2018;42:1951-62.

6. Morris MA, Wilkins E, Timmins KA, Bryant M, Birkin M, Griffiths C. Can big data solve a big problem? Reporting the obesity data landscape in line with the Foresight obesity system map. Int J Obes (Lond). 2018;42:1963-76.

7. Fuller D, Buote R, Stanley K. A glossary for big data in population and public health: discussion and commentary on terminology and research methods. J Epidemiol Community Health. 2017;71:1113-17.

8. Hughes R. Definitions for public health nutrition: a developing consensus. Public Health Nutr. 2003;6:615-20.

9. DeGregory KW, Kuiper P, DeSilvio T, Pleuss JD, Miller R, Roginski JW, et al. A review of machine learning in obesity. Obes Rev. 2018;19:668-85.

10. Sweeting HN. Measurement and definitions of obesity in childhood and adolescence: a field guide for the uninitiated. Nutr J. 2007;6:32.

11. van Mil JW, Henman M. Terminology, the importance of defining. Int J Clin Pharm. 2016;38:709-13.

12. Economic and Social Research Council. Big Data Network Phase 3: New and Emerging Forms of Data - Policy Demonstrator Projects Call specification. https://esrc.ukri.org/files/funding/ funding-opportunities/new-and-emerging-forms-of-data-policydemonstrator-projects-call-spec/. Accessed 11 Oct 2018.

13. European Commission. Horizon 2020 Work Programme 2016-2017. http://ec.europa.eu/research/participants/data/ref/h2020/wp/2016_ 2017/main/h2020-wp1617-health_en.pdf. Accessed 11 Oct 2018.

14. Cancer Research UK. Detect cancer earlier by interrogating medical and non-medical data sets using machine and deeplearning. https://www.cancerresearchuk.org/funding-for-resea rchers/how-we-deliver-research/grand-challenge-award/artificialintelligence. Accessed 11 Oct 2018.

15. Big O. Big data against childhood Obesity. https://bigoprogram. eu/. Accessed 11 Oct 2018.

16. Dickersin K. Systematic reviews in epidemiology: why are we so far behind? Int J Epidemiol. 2002;31:6-12.

17. European Parliament and Council. Regulation (EU) 2016/679 of the European Parliament and of the Council of 27 April 2016 on the protection of natural persons with regard to the processing of personal data and on the free movement of such data, and repealing Directive 95/46/EC (GeneralData Protection Regulation). Off J Eur Union. 2016;L119:1-88.

18. Information Commissioner's Office. Personal data breaches. https://ico.org.uk/for-organisations/guide-to-the-general-dataprotection-regulation-gdpr/personal-data-breaches/. Accessed 22 Oct 2018.

19. Amankwah-Amoah J. Emerging economies, emerging challenges: Mobilising and capturing value from big data. Technol Forecast Soc Change. 2016;110:167-74.

20. Vayena E, Salathe M, Madoff LC, Brownstein JS. Ethical challenges of big data in public health. PLoS Comput Biol. 2015;11: e1003904.

21. Lazer D, Radford J. Data ex machina: introduction to big data. Annu Rev Sociol. 2017;43:19-39.

22. Mooney SJ, Pejaver V. Big data in public health: terminology, machine learning, and privacy. Annu Rev Public Health. 2018;39:95-112.

23. Mai J-E. Big data privacy: The datafication of personal information. Inf Soc. 2016;32:192-99.

24. Mooney SJ, Westreich DJ, El-Sayed AM. Commentary: epidemiology in the era of big data. Epidemiology. 2015;26:390-4. 
25. Morris MA, Birkin M. The ESRC Strategic Network for Obesity: tackling obesity with big data. Int J Obes (Lond). 2018;42: 1948-50.

26. Rowe G, Wright G. The Delphi technique as a forecasting tool: issues and analysis. Int J Forecast. 1999;15:353-75.

27. Jones J, Hunter D. Consensus methods for medical and health services research. BMJ. 1995;311:376-80.

28. Byrne S, Wake M, Blumberg D, Dibley M. Identifying priority areas for longitudinal research in childhood obesity: Delphi technique survey. Pediatr Obes. 2008;3:120-22.

29. Faulkner GE, Grootendorst P, Nguyen VH, Andreyeva T, ArbourNicitopoulos K, Auld MC, et al. Economic instruments for obesity prevention: results of a scoping review and modified Delphi survey. Int J Behav Nutr Phys Act. 2011;8:109.

30. Gillis L, Tomkinson G, Olds T, Moreira C, Christie C, Nigg C, et al. Research priorities for child and adolescent physical activity and sedentary behaviours: an international perspective using a twin-panel Delphi procedure. Int $\mathrm{J}$ Behav Nutr Phys Act. 2013;10:112.

31. Huijg JM, Crone MR, Verheijden MW, van der Zouwe N, Middelkoop BJ, Gebhardt WA. Factors influencing the adoption, implementation, and continuation of physical activity interventions in primary health care: a Delphi study. BMC Fam Pract. 2013;14:142.

32. Akins RB, Tolson H, Cole BR. Stability of response characteristics of a Delphi panel: application of bootstrap data expansion. BMC Med Res Methodol. 2005;5:37.

33. Iqbal S, Pipon-Young L. The Delphi method. Psychologist. 2009;22:598-600.

34. Crane D, Henderson EJ, Chadwick DR. Exploring the acceptability of a 'limited patient consent procedure' for a proposed blood-borne virus screening programme: a Delphi consensus building technique. BMJ Open. 2017;7:e015373. https://doi.org/ 10.1136/bmjopen-2016-015373.

35. Cook S, Aikens JE, Berry CA, MoNabb WL. Development of the diabetes problem-solving measure for adolescents. Diabetes Educ. 2001;27:865-74.

36. Rose CM, Kagan AR. The final report of the expert panel for the radiation oncology bone metastasis work group of the American College of Radiology 1. Int J Radiat Oncol Biol Phys. 1998;40:1117-24.

37. Wainwright $\mathrm{P}$, Gallagher A, Tompsett $\mathrm{H}$, Atkins C. The use of vignettes within a Delphi exercise: a useful approach in empirical ethics? J Med Ethics. 2010;36:656-60.

38. Wigton RS, Darr CA, Corbett KK, Nickol DR, Gonzales R. How do community practitioners decide whether to prescribe antibiotics for acute respiratory tract infections? J Gen Intern Med. 2008;23:1615.

39. Murphy MK, Black NA, Lamping DL, McKee CM, Sanderson $\mathrm{CF}$, Askham J, et al. Consensus development methods, and their use in clinical guideline development. Health Technol Assess. 1998;2:1-88

40. Slade SC, Dionne CE, Underwood M, Buchbinder R. Standardised method for reporting exercise programmes: protocol for a modified Delphi study. BMJ Open. 2014;4:e006682.

41. Okoli C, Pawlowski SD. The Delphi method as a research tool: an example, design considerations and applications. Inf \& Manag. 2004;42:15-29.

42. Henderson EJ, Rubin GP. Development of a community-based model for respiratory care services. BMC Health Serv Res. 2012;12:193.

43. Diamond IR, Grant RC, Feldman BM, Pencharz PB, Ling SC, Moore AM, et al. Defining consensus: a systematic review recommends methodologic criteria for reporting of Delphi studies. J Clin Epidemiol. 2014;67:401-09.

44. Duffield C. The Delphi technique: a comparison of results obtained using two expert panels. Int J Nurs Stud. 1993;30:22737.

45. IBM SPSS Statistics for Windows, version 24.0 [program]. IMB, Corp., Armonk, NY; 2016.

46. University of Leeds. Consumer Data Research Centre. https://lida. leeds.ac.uk/research/consumer-data-research-centre/. Accessed 28 May 2018.

47. University of Southampton. Research Group: Administrative Data Research Centre for England. https://www.southampton.ac.uk/ s3ri/research/groups/administrative_data_research_centre_for_ england.page. Accessed 28 May 2018.

48. Roßmann B, Canzaniello A, von der Gracht H, Hartmann E. The future and social impact of Big Data Analytics in Supply Chain Management: results from a Delphi study. Technol Forecast Soc Change. 2018;130:135-49.

49. Moore GF, Audrey S, Barker M, Bond L, Bonell C, Hardeman W, et al. Process evaluation of complex interventions: Medical Research Council guidance. BMJ. 2015;350:h1258.

50. Dugan TM, Mukhopadhyay S, Carroll A, Downs S. Machine learning techniques for prediction of early childhood obesity. Appl Clin Inform. 2015;6:506-20.

51. Kaplan RM, Chambers DA, Glasgow RE. Big data and large sample size: a cautionary note on the potential for bias. Clin Transl Sci. 2014;7:342-6.

52. von Elm E, Altman DG, Egger M, Pocock SJ, Gotzsche PC, Vandenbroucke JP, et al. The Strengthening the Reporting of Observational Studies in Epidemiology (STROBE) statement: guidelines for reporting observational studies. Lancet. 2007;370:1453-7.

53. Liberati A, Altman DG, Tetzlaff J, Mulrow C, Gotzsche PC, Ioannidis JP, et al. The PRISMA statement for reporting systematic reviews and meta-analyses of studies that evaluate healthcare interventions: explanation and elaboration. BMJ. 2009;339:b2700. 